\section{Conference Report \\ The XI International AIDS Conference in Vancouver: perspectives from epidemiology and public health}

UCL Academic

Department of STD, Mortimer Market Centre, London WC1E 6AE, UK

K A Fenton

Accepted for publication 19 August 1996

\author{
K A Fenton
}

Advances and reports in epidemiology and public health at the XI International AIDS Conference were covered in Track C. Under the conference theme of One World. One Hope, the oral and poster presentations provided a dedicated forum for discussion on HIV/AIDS epidemiology, natural history and public health strategies including education, prevention, screening, partner notification and surveillance. A wide range of areas was covered which illustrated the diversity and creativity employed in public health and prevention activities. This report will focus on the major themes which arose from seven main sub-categories within this Track: Descriptive epidemiology; HIV transmission incidence and risk; Natural history and progression; HIV/STD interaction; Prevention issues; Public Health activities and Methodologic issues.

\section{Descriptive epidemiology}

Many of the oral and poster presentations throughout the conference focused on the descriptive epidemiology of HIV/AIDS, defining at risk populations, prevalence of risk behaviours and future trends for the epidemic. There was a growing realisation that the HIV pandemic is now composed of distinct epidemics each with its own features and force, and disproportionately impacting on the developing world. As such, the participation of developing country states in the oral and plenary sessions was highly visible and forceful, serving to focus participants on key issues.
Cumulatively, since the beginning of the epidemic until mid-1996, an estimated 27.9 million people have been infected with HIV and more than 6 million adults have developed AIDS. ${ }^{1}$ The global cumulative number of HIV infections has more than doubled since the beginning of the decade, from about 10 million in 1990 to 25.5 million by mid 1996 . In mid-July 1996, an estimated 21.8 million adults and children worldwide were living with HIV/AIDS (figure), of whom 20.4 million (94\%) were in the developing world. Close to 19 million adults and children $(86 \%$ of the world total) were living in with HIV/AIDS in sub-Saharan Africa, South and South East Asia.

Developed countries: The growth of the AIDS epidemic in North America and many northern parts of Western Europe has slowed in recent years and is approaching a level incidence, largely owing to the decline in sexual transmission between men. The increase in AIDS incidence in the 1990s in North America has been greatest for women, ethnic minorities and infections occurring through heterosexual transmission. In the USA there has been an annual decline in AIDS incidence among children less than 13 years of age, which may reflect lower conception rates in women diagnosed with HIV and the possible impact of maternal and neonatal zidovudine (ZDV) therapy on HIV transmission. In contrast, in the countries of the southern part of western Europe there is no indication of the
Estimated number of
persons living with persons living with 1996.
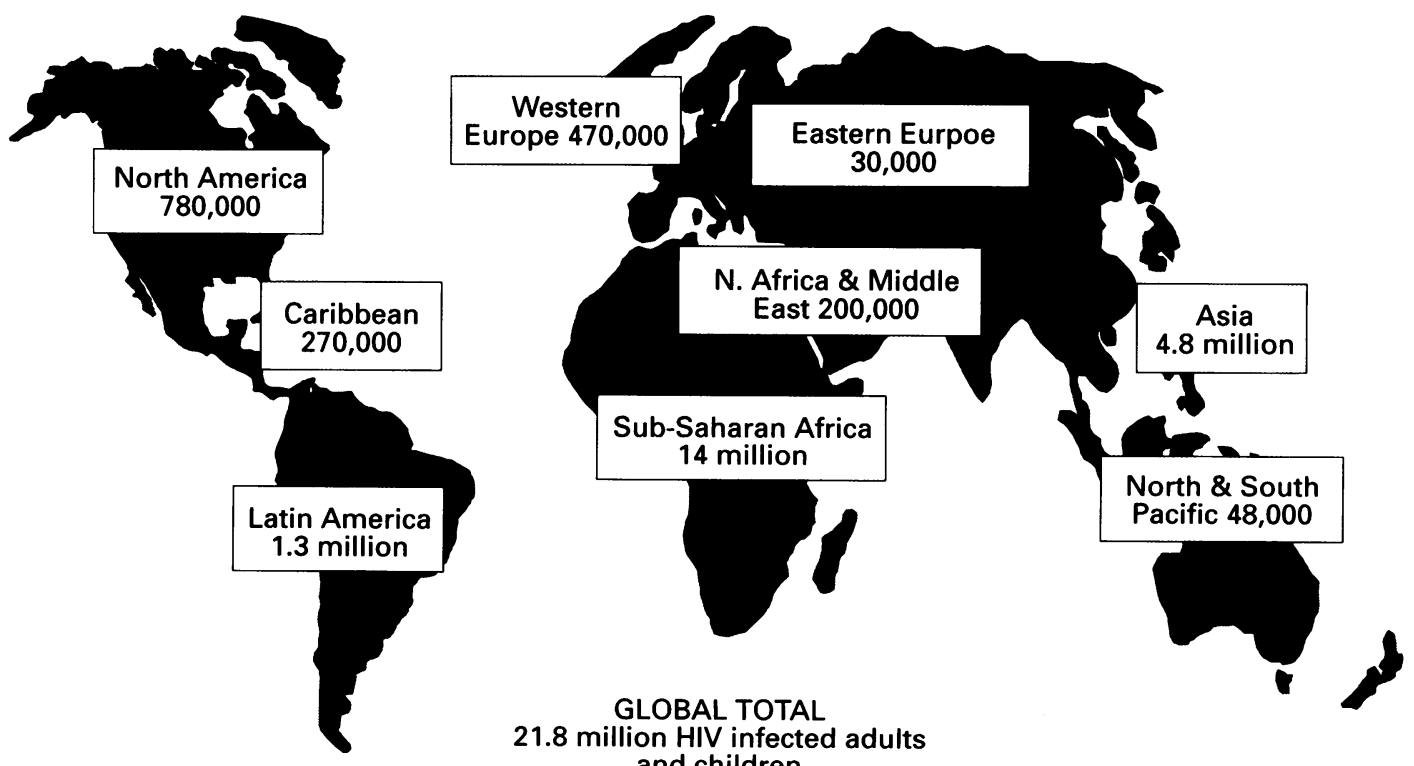

GLOBAL TOTAL

21.8 million HIV infected adults and children 
AIDS epidemic levelling off. In central and eastern Europe (with the exception of Romania) the HIV/AIDS epidemic is much more recent and AIDS incidence is lower than in Western Europe.

Developing countries: In sub-Saharan Africa, by mid-1996, 13.3 million adults were living with HIV, representing $60 \%$ of the world's total. In Kenya, Malawi, Rwanda, Uganda, Zambia and Zimbabwe more than $10 \%$ of women attending antenatal clinics surveyed in urban areas have been found to be HIV infected, with rates which may exceed $40 \%$ in some surveillance sites. In these populations, transmission of HIV occurs mainly through sexual contact, beginning early in the teens and peaking before age 25 years. HIV epidemics have recently expanded in the southern African states. The spread of HIV-2 has remaind rather stable in West Africa with the highest prevalence being reported in Guinea Bissau and Southern Senegal.

The Asian epidemic is still in its early phase and has to date been one of extreme diversity ranging from countries with low HIV prevalence rates (for example Mongolia, DPR Korea) to those with high prevalence (Cambodia, Myanmar, Thailand). In south and southeast Asia it is estimated that 5 million people have been infected with HIV and 0.33 million adults have to date developed AIDS. In Thailand there are an estimated 750000 people living with HIV predominantly in the intravenous drug user (IDU) population. By 1994, seroprevalence rates of $35 \%$ among IVDUs, $8.6 \%$ for STD clinic attendees and $33 \%$ among brothel based sex workers were reported. In India, with an estimated 1.75 million HIV infections at the end of 1994 , there is great geographical variation with higher seroprevalences being recorded in the South and Western parts of the country.

As of mid-1996, Latin America and the Caribbean accounted for $13.4 \%$ of the cases reported worldwide to the WHO. Some countries are at particular risk of rapid dissemination of HIV from traditional at risk groups (such as commercial sex workers, men who have sex with men) to other vulnerable groups in the population (that is, women, youth, children). Sexual transmission of HIV/AIDS accounts for $80 \%$ of overall transmission in the region ranging from $64 \%$ in Brazil to as high as $93 \%$ in the Andean subregion (Bolivia, Colombia, Ecuador, Peru, Venezuela).

Explosive epidemics: There are areas of rapid spread of the virus, as in India, Cambodia and South Africa. Throughout the conference participants were constantly reminded of the need for continued vigilance for those countries and areas with the potential for explosive epidemics such as Indonesia, Myanmar, and several countries in West Africa. In many eastern European countries, although a rapid spread of HIV has not yet been seen, there is concern because of an alarming increase in STDs due to migration, increase in commercial sex workers and deteriorating health services.
HIV transmission, incidence and risk

Men who have sex with men (MSM): Although the epidemic among MSM remains the predominant mode of transmission within many industrialised countries, there is increasing evidence to suggest a levelling of the HIV/AIDS incidence rates among this group as a whole. There remains much concern, however, that young and ethnic minority MSM may continue to be at high risk. Particular concerns with young gay men include higher sensation seeking personality dispositions, larger number of unprotected sexual partners, substance use/abuse during sex. Among ethnic minority MSM specific concerns surround the prevalence of high risk behaviours and misconceptions surrounding risk behaviour.

Heterosexual transmission: Women are increasingly becoming infected with HIV and at an earlier age than men. As of late 1995, almost 9 million women had been infected with HIV since the start of the epidemic. In many industrialised countries, HIV/AIDS is now disproportionately affecting women from ethnic minority backgrounds. In the US, AIDS incidence is rising fastest among women, particularly black women and increasingly reflects heterosexual transmission of HIV. The role of HIV subtypes, male circumcision, coincident STDs, the stage of HIV infection in influencing heterosexual transmission of the virus were reinforced by a number of systematic review presentations..$^{234}$

IDUs: There is continued global diffusion of injecting drug use. By 1996 IDU was reported from 121 countries with 81 having IDU-HIV infections. IDU behaviour may be spread by wars and other conflicts, changes in the nature and distribution of drug production, drug trafficking routes; patterns of regional communication. ${ }^{5}$ This is of particular relevance to the southern West European countries, North America and South and Southeast Asia. New infections continue to occur in spite of intensive health promotional campaigns. Risk of infection is higher soon after starting drug injection, ${ }^{67}$ within IDU social networks than across networks with strangers, ${ }^{89}$ and confounded by the growing heterosexual HIV epidemic $^{1011}$ in many states.

Vertical transmission: There were initial indications that different subtypes may also play a role in the efficacy of transmission of HIV ( $E$ being more effectively transmitted than B). The effect of AZT in reducing the probability of transmission was convincingly shown in a variety of observational and controlled studies. ${ }^{121314}$ The role of Caesarean section in reducing transmission is becoming less controversial with an RCT of interventions (AZT, elective Caesarean section, both or none) in Switzerland indicating an additive protective effect of both zidovudine prophylaxis and elective Caesarean section. ${ }^{15}$ The breast feeding debate continued with Grey et al in Soweto ${ }^{16}$ showing significantly reduced transmission of HIV among formula fed infants in their cohort 
with no additional increase in infant mortality. There remains a need for further studies in this area.

\section{Natural history and progression}

There were many presentations examining cofactors in disease progression including HLA typing (HLA CW ${ }^{\star} 0602$, B27 associated with delayed progression) ${ }^{17}$; serum $\mathrm{B} 12$ and vitamin $\mathrm{E},{ }^{18} \mathrm{IgA},{ }^{19} \mathrm{HIV} \mathrm{RNA}^{20}$ and fever alone, during seroconversion. ${ }^{21}$ Disease progression in gay men appears to be more rapid than in heterosexual men and women ${ }^{22}$ and this could be explained by coinfections, phenotypic and genotypic viral characteristics. Gay men are also at increased risk of non Hodgkins lymphoma and Hodgkins disease. ${ }^{23}$ Among haemophilic men, $\mathrm{Sabin}^{24}$ reported a median time to AIDS of 14 years.

\section{HIVISTD interaction}

The importance of genital secretions associated with STDs and the efficacy of transmission of HIV were underscored with presentations showing increased HIV RNA load in genital secretions of women with cervicitis, in men with urethritis, and immunosuppressed patients. The impact of treatment of genital infections in reducing the viral RNA load in the semen ${ }^{25}$ and in genital secretions was presented. Evaluations of different strategies for STD management were presented which included improving STD services, ${ }^{26}$ mass treatment programmes, ${ }^{27}$ and syndromic STD management. ${ }^{2628}$ The importance of recognising asymptomatic genital infections as a reservoir for repeat infection was underscored.

\section{Prevention issues}

Realising the limited effectiveness of current safer sex health promotion messages, there is now an increasing move towards developing more creative and sophisticated interventions with gay men that are flexible and responsive to the changing patterns of gay male sexual behaviour. Personal strategies for promoting safer sex with gay men, ${ }^{29}$ placing the responsibility for safer sex on both partners instead of individuals and the responsibility of gay establishments ${ }^{30}$ in promoting safer sex among their clients were key themes.

Among heterosexuals, prevention activities remain focused around strategies to improve condom use for casual and new partnerships. Vaginal viricides were increasingly promoted as a means of women protecting themselves against HIV, although wider issues of empowering women through education, information and employment were stressed. Among IDUs, the real advantages of needle exchange programmes and alternative strategies for reducing HIV transmission within the IDU population were debated. There is now convincing evidence to suggest that needle exchange programmes do not increase drug use and may attract IDUs at increased risk of HIV. ${ }^{31}{ }^{32}$

\section{Public health activities}

Many descriptive studies and short term evaluations were presented under the theme of education and awareness. They were mainly knowledge and attitude based with few utilising behavioural outcomes or employing a randomised controlled trial methodology. Key strategies included use of peer-based techniques, social marketing and using persons living with AIDS in prevention efforts. Innovative interventions using communitybased techniques predominated the poster presentations. The debate on partner notification for HIV as a public health strategy occurred throughout the conference. Opponents question the validity of utilising a strategy of questionable efficacy and with potential adverse emotional and social sequelae, whereas proponents stress the need for training and sensitivity in achieving goals. Outreach initiatives to try to reach groups such as IDUs, the homeless, were presented from the perspectives of both developing and developed countries. The importance of programme planning and management as applicable to community based groups and non-governmental organisations (NGOs) fighting against AIDS were emphasised. There were also calls for continuous review and appraisal of government HIV prevention strategies.

\section{Methodologic issues}

Work on HIV vaccines is underway in Thailand and results of phase I and II trials were presented..$^{33}$ The results so far are encouraging and suggest that there are populations in which vaccine feasibility trials may be carried out and that the side effect profiles of the vaccines are acceptable. More work remains on vaccine efficacy and determining the appropriate populations for use. ${ }^{34}$ Developments in technologies available for diagnosing HIV were presented (for example, blood spot, urine and salivary testing) which may have a role in HIV field surveillance. Home testing technology is expanding despite the ethical and practical dilemmas it presents, and a revision of old misgivings will be increasingly needed.

In conclusion, Track $C$ illustrated the varied and creative epidemiological and public health strategies being employed in the fight against AIDS. In many instances simple public health interventions were effective in bringing about change. This was regardless of resources available, but depended upon proper management, with the commitment and involvement of the community, politicians and health care professionals. Perhaps this is the one hope.

Abstracts for references 2-34 are contained in the XI International AIDS Conference Abstract Manuals, Vols I and II.

\footnotetext{
1 Final report of the XI International Conference Status and Trends of the Global HIV/AIDS Pandemic Satellite Trends of the Global HIVIAIDS Pandemic Satellite Prevention (AIDSCAP) Project of Family Health
} 
International, the Francois-Xavier Bagnoud Center for Health and Human Rights of the Harvard School of Public Health and the Joint United Nations Programme on HIV/AIDS (UNAIDS).

2 Moses S. Association between lack of male circumcision and risk for HIV infection: Review of the epidemiological evidence. Oral We.C452.

3 Wasserheit J. Heterogeneity of heterosexual transmission: The role of other STDs. Oral We.C453.

4 De Vincenzi I. Infectiousness and stage of HIV infection. Oral We.C454.

5 Stimpson G. Continued global diffusion of Injecting Drug Oreal has major in

6 Zaccarelli M. Understanding the dynamics of HIV infection among IDU in Rome in the 1990s: The combined tion among
use of crossectional longitudinal and behavioural data. use of crossection

7 Doherty MC. Younger age of initiating injecting drug use is associated with risks behaviours and HIV infection in short-term injectors. Oral Mo.C464.

8 Neaigus A. Drug injectors' social networks can be risk factors for syringe sharing. Oral Mo.C462.

9 Lamothe F. Risk factors for HIV seroconversion among injecting drug users in the Saint-Luc Cohort, Montreal 1988-1995. Poster Tu.C2502.

10 Mayne T. High risk of HIV and STD transmission among HIV positive IDUS in southern France. Poster Tu.C2508.

11 Sharma Arun Kumar. Prevalence of HIV related risk behaviour among IDU in a resettlement colony in Delhi. Poster Tu.C2511.

12 Simonds RJ. Declining mother-to-child HIV transmission following perinatal zidovudine recommendations, United following perinatal zid
States. Oral Tu.C440.

13 Mansergh G. Cost effectiveness of short course Zidovudine to prevent perinatal HIV in Developing country settings. Oral Th.C413.

14 Ekpini ER. Safety of and adherence to oral Zidovudine administered in late pregnancy to HIV-1 infected pregnant women in Abidjan, Cote D'Ivoire. Oral Th.C412.

15 Kind C. Effects of Zidovudine prophylactics and elective Cesarean section on vertical HIV transmission. Ora Tu.C442.

16 Gray G. The effect of breastfeeding on vertical transmission of HIV-1 in Soweto, South Africa. Oral Th.C415.

17 Easterbrook PJ. HLA Class 1 genes and long-term progression in HIV infection. Oral Tu.C550.

18 Tang A. The role of serum micronutrient levels in HIV-1 disease progression. Oral Mo.C320.

19 Shinobu T. Fraction of patients free of clinical AIDS after CD4 cell count dropping less than $200 \times 10^{6} / 1$ in Japanese hemophiliacs infected with HIV-1. Oral Japanese
Mo.C321.

20 O'Brien TR. HIV-1 RNA levels in early chronic infection: Association with AIDS and long term non progression. Oral Mo.C323.

21 Veugelers $P$. Incidence and Prognostic significance of symptomatic primary HIV-1 infection in homosexual men. Oral Mo.C324.

22 Meyer L. Comparison of HIV disease progression among homosexual men, heterosexual men and heterosexual women. Oral Tu.C430.

23 Hessol N. Increased incidence of cancer among homosexual men, New York city and San Francisco, 1978-1990. Oral Tu.C433.

24 Sabin C. The progression of HIV infection in a cohort of hemophilic men followed up to 16 years after seroconversion. Oral Tu.C432.

25 Hoffman I. Effects of urethritis therapy on the concentration of HIV-1 in seminal plasma. Oral Mo.C903.

26 Hayes R. Cost effectiveness of Improved STD Treatment services as a preventive intervention against HIV in Mwanza region, Tanzania. Oral Mo.C444.

27 Wawer M. Community based trial of mass STD treatment for HIV control, Rakai, Uganda: Preliminary data on STD declines. Oral C443.

28 Ettiegene-Traore V. HIV seroincidence and STD prevalence during an intervention study among female sex workers in Abidjan, Cote D'Ivoire: Preliminary findings. Oral Mo.C442

29 Kinder P. A new prevention education strategy for gay men: Responding to the impact of AIDS on gay men's lives. Oral We C442.

30 Fleutelot E. A charter defining the responsibilities of gay businesses that provide opportunities for sexual contact. Oral We.C444

31 Alary M. Incidence and prevalence of HIV and risk behaviours among IDU attending needle exchange programs in Quebec. Oral Tu.C321.

32 Archibald C. Needle Exchange Program attracts high-risk injecting drug users. Oral C 320

33 Migasena S. A phase I/II trial of MNRGP $120 \mathrm{HIV}-1$ vaccine (Genentech, Inc.) among injecting drug users (IDUs) in Bangkok, Thailand: Safety and immunogenicity. Oral We.C460.

34 Osmanov S. HIV vaccine trials in developing countriesThe UNAIDS perspective. Oral We.C463. 